\title{
Observation of inertial energy cascade in interplanetary space plasma
}

\author{
Luca Sorriso-Valvo ${ }^{1}$, Raffaele Marino ${ }^{2}$, Vincenzo Carbone ${ }^{2}$, \\ Fabio Lepreti ${ }^{2}$, Pierluigi Veltri ${ }^{2}$, Alain Noullez ${ }^{3}$, \\ Roberto Bruno ${ }^{4}$, Bruno Bavassano ${ }^{4}$, Ermanno Pietropaolo ${ }^{5}$
}

${ }^{1}$ Licryl Regional Laboratory - INFM/CNR, Ponte P. Bucci, Cubo 33C, 87036 Rende (CS), Italy

2 Dipartimento di Fisica, Università della Calabria, and CNISM,

Ponte P. Bucci Cubo 31C, 87036 Rende (CS), Italy

3 Observatoire de la Côte d'Azur,

Boulevard de l'Observatoire, 06304 Nice, France

${ }^{4}$ IFSI/INAF, Via Fosso del Cavaliere, 00133 Roma, Italy

${ }^{5}$ Dipartimento di Fisica, Università dell'Aquila,

Via Vetoio, 67010, L'Aquila, Italy

January 29, 2018 


\begin{abstract}
We show in this article direct evidence for the presence of an inertial energy cascade, the most characteristic signature of hydromagnetic turbulence (MHD), in the solar wind as observed by the Ulysses spacecraft. After a brief rederivation of the equivalent of Yaglom's law for MHD turbulence, we show that a linear relation is indeed observed for the scaling of mixed third order structure functions involving Elsässer variables. This experimental result, confirming the prescription stemming from a theorem for MHD turbulence, firmly establishes the turbulent character of low-frequency velocity and magnetic field fluctuations in the solar wind plasma.
\end{abstract}


Space flights have shown that the interplanetary medium is permeated by a supersonic, highly turbulent plasma flowing out from the solar corona, the so called solar wind [1, 2]. The turbulent character of the flow, at frequencies below the ion gyrofrequency $f_{c i} \simeq 1 \mathrm{~Hz}$, has been invoked since the first Mariner mission [3]. In fact, velocity and magnetic fluctuations power spectra are close to the Kolmogorov's $-5 / 3$ law [2, 6]. However, even if fields fluctuations are usually considered within the framework of magnetohydrodynamic (MHD) turbulence 2], a firm established proof of the existence of an energy cascade, namely the main characteristic of turbulence, remains a conjecture so far [4]. This apparent lack could be fulfilled through the evidence for the existence of the only exact and nontrivial result of turbulence [6], that is a relation between the third order moment of the longitudinal increments of the fields and the separation [5]. This observation would firmly put low frequency solar wind fluctuations within the framework of MHD turbulence. The importance of such question stands beyond the understanding of the basic physics of solar wind turbulence. For example, it is well known that turbulence is one of the main obstacles to the confinement of plasmas in the fusion devices [7, 8]. The understanding of interplanetary turbulence and its effects on energetic particle transport is of great importance also for Space Weather research [9], which is a relevant issue for spacecrafts and communication satellites operations, and for the security of human beings. Finally, more theoretical problems are concerned, such as the puzzle of solar coronal heating due to the turbulent flux toward small scales [10].

Incompressible MHD equations are more complicated than the standard neutral fluid mechanics equations because the velocity of the charged fluid is coupled with the magnetic field generated by the motion of the fluid itself. However, written in terms of the Elsässer variables defined as $\boldsymbol{z}^{ \pm}=$ $\boldsymbol{v} \pm(4 \pi \rho)^{-1 / 2} \boldsymbol{b}(\boldsymbol{v}$ and $\boldsymbol{b}$ are the velocity and magnetic field respectively and $\rho$ the mass density), they have the same structure as the Navier-Stokes equations [4]

$$
\partial_{t} \boldsymbol{z}^{ \pm}+\boldsymbol{z}^{\mp} \cdot \boldsymbol{\nabla} \boldsymbol{z}^{ \pm}=-\nabla P / \rho+\left(\frac{\nu+\kappa}{2}\right) \nabla^{2} \boldsymbol{z}^{ \pm}+\left(\frac{\nu-\kappa}{2}\right) \nabla^{2} \boldsymbol{z}^{\mp}
$$

where $P$ is the total hydromagnetic pressure, while $\nu$ is the viscosity and $\kappa$ the magnetic diffusivity. In particular, the nonlinear term appears as $\boldsymbol{z}^{\mp} \cdot \boldsymbol{\nabla} \boldsymbol{z}^{ \pm}$, suggesting the form of a transport process, in which Alfvénic MHD fluctuations $\boldsymbol{z}^{ \pm}$propagating along the background magnetic field are transported by fluctuations $\boldsymbol{z}^{\mp}$ propagating in the opposite direction. This transport is active as $\boldsymbol{z}^{ \pm}$and $\boldsymbol{z}^{\mp}$ are clearly not independent. Still, following the same procedure as in [11, 12, and assuming local homogeneity, a relation similar to the Yaglom equation for the transport of a passive quantity [13] can be 
obtained in the stationary state

$$
\begin{aligned}
\partial_{\|} Y^{ \pm}(r)= & -\frac{4}{3} \epsilon^{ \pm}+2 \nu \nabla^{2}\left\langle\left|\Delta \boldsymbol{z}^{ \pm}\right|^{2}\right\rangle \\
& -2\left\langle\Delta \boldsymbol{z}^{ \pm} \cdot\left(\boldsymbol{\nabla}+\boldsymbol{\nabla}^{\prime}\right) \Delta P / \rho\right\rangle+\left\langle\boldsymbol{z}^{\mp} \cdot\left(\boldsymbol{\nabla}+\boldsymbol{\nabla}^{\prime}\right)\left|\Delta \boldsymbol{z}^{ \pm}\right|^{2}\right\rangle .
\end{aligned}
$$

Here, $\Delta \boldsymbol{z}^{ \pm} \equiv \boldsymbol{z}^{ \pm}\left(\boldsymbol{x}^{\prime}\right)-\boldsymbol{z}^{ \pm}(\boldsymbol{x})$ are the (vector) increments of the fluctuations between two points $\boldsymbol{x}$ and $\boldsymbol{x}^{\prime} \equiv \boldsymbol{x}+\boldsymbol{r}, \boldsymbol{\nabla}$ and $\boldsymbol{\nabla}^{\prime}$ are the gradients at the corresponding two points, $\partial_{\|}$is the longitudinal derivative along the separation $\boldsymbol{r}$, while $Y^{ \pm}(r)$ are the mixed third order structure function $\left\langle\left|\Delta \boldsymbol{z}^{ \pm}\right|^{2} \Delta z_{\|}^{\mp}\right\rangle$ and $\epsilon^{ \pm} \equiv \nu\left\langle\left|\boldsymbol{\nabla} \boldsymbol{z}^{ \pm}\right|^{2}\right\rangle \stackrel{\text { hom }}{=} 3 \nu\left\langle\left|\partial_{\|} \boldsymbol{z}^{ \pm}\right|^{2}\right\rangle$ are the pseudo-energy average dissipation rates, namely the dissipation rates of both $\left\langle\left|\boldsymbol{z}^{ \pm}\right|^{2}\right\rangle / 2$ respectively. Finally, $\Delta P$ represent the increment of the total pressure fluctuations and the kinematic viscosity $\nu$ is here assumed to be equal to the magnetic diffusivity $\kappa$ (this last assumption is in fact not necessary if we concentrate on the inertial range, as we will do from now on).

The last term on the r.h.s. of equation (2) is related to large-scale inhomogeneities and disappears if the flow is globally homogeneous. Also, assuming local isotropy, the term containing pressure correlation vanishes, so that after longitudinal integration of (21) and in the inertial range of MHD turbulence (i.e. when $\nu \rightarrow 0$ ), a linear scaling law

$$
Y^{ \pm}(r)=-\frac{4}{3} \epsilon^{ \pm} r
$$

is obtained, characterizing a turbulent cascade with a well-defined finite energy flux $\epsilon^{ \pm}$. An alternative derivation of this result using correlators instead of structure functions was also obtained in [14], and observed in numerical simulations [15]. When neutral fluid turbulence is considered, equation (3) becomes [1] $\left\langle|\Delta \boldsymbol{v}|^{2} \Delta v_{\|}\right\rangle=-4 / 3 \epsilon r$ ( $\epsilon$ being the average kinetic energy dissipation rate), from which Kolmogorov's $-4 / 5$ law for the longitudinal third order structure function can be recovered if there is full isotropy as $\left\langle\left(\Delta v_{\|}\right)^{3}\right\rangle=-4 / 5 \epsilon r$.

In this work, we show that relation (3) is indeed satisfied in some periods within solar wind turbulence. In order to avoid variations of the solar activity and ecliptic disturbances (like slow wind sources, Coronal Mass Ejections, ecliptic current sheet, and so on), we use high speed polar wind data measured by the Ulysses spacecraft [16, 17]. In particular, we analyse here the first seven months of 1996, when the heliocentric distance slowly increased from $3 \mathrm{AU}$ to $4 \mathrm{AU}$, while the heliolatitude decreased from about $55^{\circ}$ to $30^{\circ}$. The fields components are given in the $R T N$ reference frame, where $R$ (radial) indicates the sun-spacecraft direction, centered on 
the spacecraft and pointing out of the sun, $N$ (normal) lies in the plane containing the radial direction and the sun rotation axis, while $T$ completes the right-handed reference frame. Note that, since the wind speed in the spacecraft frame is much larger than the typical velocity fluctuations, and is nearly aligned with the $R$ radial direction, time fluctuations are in fact spatial fluctuations with time and space scales ( $\tau$ and $r$ respectively) related through the Taylor hypothesis, so that $r=-\left\langle v_{R}\right\rangle \tau$ (note the reversed sign). From the 8 minutes averaged time series $\boldsymbol{z}^{ \pm}(t)$, we compute the time increments $\Delta \boldsymbol{z}^{ \pm}(\tau ; t)=\boldsymbol{z}^{ \pm}(t+\tau)-\boldsymbol{z}^{ \pm}(t)$, and obtain the mixed third order structure function $Y^{ \pm}\left(-\left\langle v_{R}\right\rangle_{t} \tau\right)=\left\langle\left|\Delta \boldsymbol{z}^{ \pm}(\tau ; t)\right|^{2} \Delta z_{R}^{\mp}(\tau ; t)\right\rangle_{t}$ using moving averages $\langle\bullet\rangle_{t}$ on the time $t$ over periods spanning around 10 days, during which the fields can be considered stationary.

A linear scaling $Y^{ \pm}(\tau)=4 / 3 \epsilon^{ \pm}\left\langle v_{R}\right\rangle_{t} \tau$ is indeed observed in a significant fraction of the periods we examined, with an inertial range spanning as much as two decades, indicating the existence of a well defined inertial energy cascade range in plasma turbulence. In fact, solar wind inertial ranges can even be larger than the ones reported for laboratory fluid flows [12], showing the robustness of this result. This is the first experimental validation of the turbulence MHD theorem discussed above. Figure 1 shows an example of scaling and the extension of the inertial range, for both $Y^{ \pm}(\tau)$. The linear scaling law generally extends from a few minutes to one day or more. This happens in about 20 periods of a few days in the 7 months considered. Several other periods are found in which the scaling range is considerably reduced. In particular, the sign of $Y^{ \pm}(\tau)$ is observed to be either positive or negative. Since pseudo-energies dissipation rates are positive defined, a positive sign for $Y^{ \pm}(\tau)$ (negative for $Y^{ \pm}(r)$ ) indicates a (standard) forward cascade with pseudo-energies flowing towards the small scales to be dissipated. On the contrary, a negative $Y^{ \pm}(\tau)$ is the signature of an inverse cascade where the energy flux is being transferred on average toward larger scales. Figure 2 shows the location of the most evident scaling intervals, together with the values of the flux rate $\epsilon^{ \pm}$estimated through a fit of the scaling law (3), typically of the order of a few hundreds in $\mathrm{J} \mathrm{kg}^{-1} \mathrm{~s}^{-1}$. For comparison, values found for ordinary turbulent fluids are $1 \div 50 \mathrm{~J} \mathrm{~kg}^{-1} \mathrm{~s}^{-1}$ [18].

It is worth noting that, in a large fraction of cases, both $Y^{ \pm}(\tau)$ switch from positive to negative linear scaling (or viceversa) within the same time period when going from small to large scales (see Figure 3). The occurrence of both kind of cascades within the same flow is not so uncommon within hydrodynamic turbulence. This phenomenon has been attributed to some large scale instability, as observed for example in geophysical flows or when the flow is affected by a strong rotation [19]. In the case of solar wind plasma a possible explanation for the inverse cascade could be the enhanced 
intensity of the background magnetic field. This would make the turbulence mainly bidimensional allowing for an inverse cascade as observed in numerical simulations [20]. It should also be noticed that in most of the cases the time scale at which the cascade reverses its sign is of the order of 1 day. This scale roughly indicates where the small scale Alfvénic correlations between velocity and magnetic field are lost [21, 22. This could mean that the nature of the fluctuations changes across the break. However, these particular aspects still deserve to be adequately considered within the solar wind context.

At this point, the question is why the scaling is not observed all the time within the solar wind. As already stated, equation (2) reduces to the linear law (3) only when local homogeneity, incompressiblity and isotropy conditions are satisfied. In general, solar wind inhomogeneities play a major role at large scales so that local homogeneity is generally fulfilled within the range of interest. Regarding incompressibility, it has been shown that compressive phenomena mainly affect shocked regions and dynamical interaction regions like stream-stream interface [1, 2]. However, the time interval we analyze, because of Ulysses high latitude location, is not affected by these compressive phenomena [23]. On the other hand, it has also been shown [2] that magnetic field compressibility increases mainly at very small scales within the fast wind regime. It follows that the incompressibility assumption can be considered valid to a large extent for the analyzed interval and at intermediate scales. The large scale anisotropy, mainly due to the average magnetic field, is only partially lost at smaller scales, and a residual anisotropy is always present [24, 25], generally breaking the local isotropy assumption. Thus, while inhomogeneity, compressibility and anisotropy could all be responsible for the loss of linear scaling, anisotropy probably is the main candidate within high latitude regions of the solar wind.

It is important to note that the presence of a Yaglom-like law that involves the third order mixed moment, is more general than the phenomenology usually involving the second order moment. Indeed, while the Yaglom MHD relation (2) involves only differences along the parallel direction, that are in fact the only quantities accessible from single satellite measurements, phenomenological arguments involve the full spatial dependence of vector fields that cannot be directly measured yet. This means that our result is compatible with both Kolmogorov and Iroshnikov-Kraichnan type cascade [4, 2], and does not help in discriminating between these phenomenologies [26, 27].

In conclusion, we observed, for the first time in the solar wind, the only natural plasma directly accessible, evidence of Yaglom MHD scaling law indicating the existence of a local energy cascade in hydromagnetic turbulence. The scaling holds in a number of relatively long periods of about 10 days, and also provides the first estimation of the pseudo-energy dissipation rate. 
Although our data might not fully satisfy requirements of homogeneity, incompressibility and isotropy everywhere, the observed linear scaling extends on a wide range of scales and appears very robust. The unexpected existence of the scaling law in anisotropic, weakly compressible and inhomogeneous turbulence still needs to be fully understood. Our result estabilishes a firm point within solar wind phenomenology, and, more generally, provides a better knowledge of plasma turbulence, carrying along a wide range of practical implications on both laboratory fusion plasmas and space physics. 


\section{Bibliography}

[1] C.-Y. Tu, E. Marsch, Space Sci. Rev. 73, 1 (1995)

[2] R. Bruno, V. Carbone, Living Rev. Solar Phys. 2, 4 (2005)

[3] P.J. Coleman, Astrophys. J. 153, 317 (1968)

[4] M. Dobrowolny, A. Mangeney, P. Veltri, Phys. Rev. Lett. 45, 144 (1980)

[5] A.N. Kolmogorov, Dokl. Akad. Nauk. SSSR 30, 301 (1941)

[6] U. Frisch, Turbulence: The legacy of A.N. Kolmogorov, Cambridge Univ. Press (1995)

[7] J. Sheffield, Rev. Mod. Phys. 66, 1015 (1994)

[8] K. Krushelnick and S. Cowley, Science 309, 1502 (2005)

[9] M. Kumar, Space Weather 4, 508004 (2006)

[10] V.M. Nakariakov, L. Ofman, E.E. De Luca, B. Roberts, and J.M. Davila, Science 285, 862 (1999)

[11] R.A. Antonia, M. Ould-Rouis, F. Anselmet, Y. Zhu, J. Fluid Mech. 332, 395 (1997)

[12] L. Danaila, F. Anselmet, T. Zhou, R.A. Antonia, J. Fluid Mech. 430, 87 (2001)

[13] A.S. Monin, A.M. Yaglom, Statistical Fluid Mechanics, Vol 2, MIT Press (1975)

[14] H. Politano, A. Pouquet, Geophys. Res. Lett. 25, 273 (1998)

[15] L. Sorriso-Valvo, V. Carbone, A. Noullez, H. Politano, A. Pouquet, and P. Veltri Phys. Plasmas 9, 89 (2002) 
[16] E.J. Smith, R.G. Marsden, and D.E. Page, Science 268, 1005 (1995)

[17] A. Balogh, D.J. Southwood, R.J. Forsyth, T.S. Horbury, E.J. Smith, and B.T. Tsurutani, Science 268, 1007 (1995)

[18] S. Cerutti and C. Meneveau, Phys. Fluids 12, 1143 (2000)

[19] C. Morize, F. Moisy, M. Rabaud, Phys. Fluids 17, 095105 (2005)

[20] C.N. Baroud, B.B. Plapp, Z.-S. She, H.N. Swinney, Phys. Rev. Lett. 88, $114501(2002)$

[21] R. Bruno, B. Bavassano and U. Villante, J. Geophys. Res., 90, 4373, (1985)

[22] B.E. Goldstein et al., Geophys. Res. Lett., 22, 3393, (1995)

[23] J.T. Gosling et al., Space Sci. Rev., 72, 99, (1995)

[24] T.S. Horbury, A. Balogh, J. Geophys. Res. 106, 929 (2001)

[25] L. Sorriso-Valvo, V. Carbone, R. Bruno and P. Veltri, Europhys. Lett. $\mathbf{7 5}, 832(2006)$

[26] P. Goldreich, S. Sridhar, Astrophys. J. 438, 763 (1995)

[27] W.-C. Müller, D. Biskamp, R. Grappin, Phys. Rev. E 67, 066302 (2003)

Acknowledgments The use of data of the plasma analyzer (principal investigator D. J. McComas, Southwest Research Institute, San Antonio, Texas, USA) and of the magnetometers (principal investigator A. Balogh, The Blackett Laboratory, Imperial College, London, UK) aboard the Ulysses spacecraft is gratefully acknowledged. The data have been made available through the World Data Center A for Rockets and Satellites (NASA/GSFC, Greenbelt, Maryland, USA). 

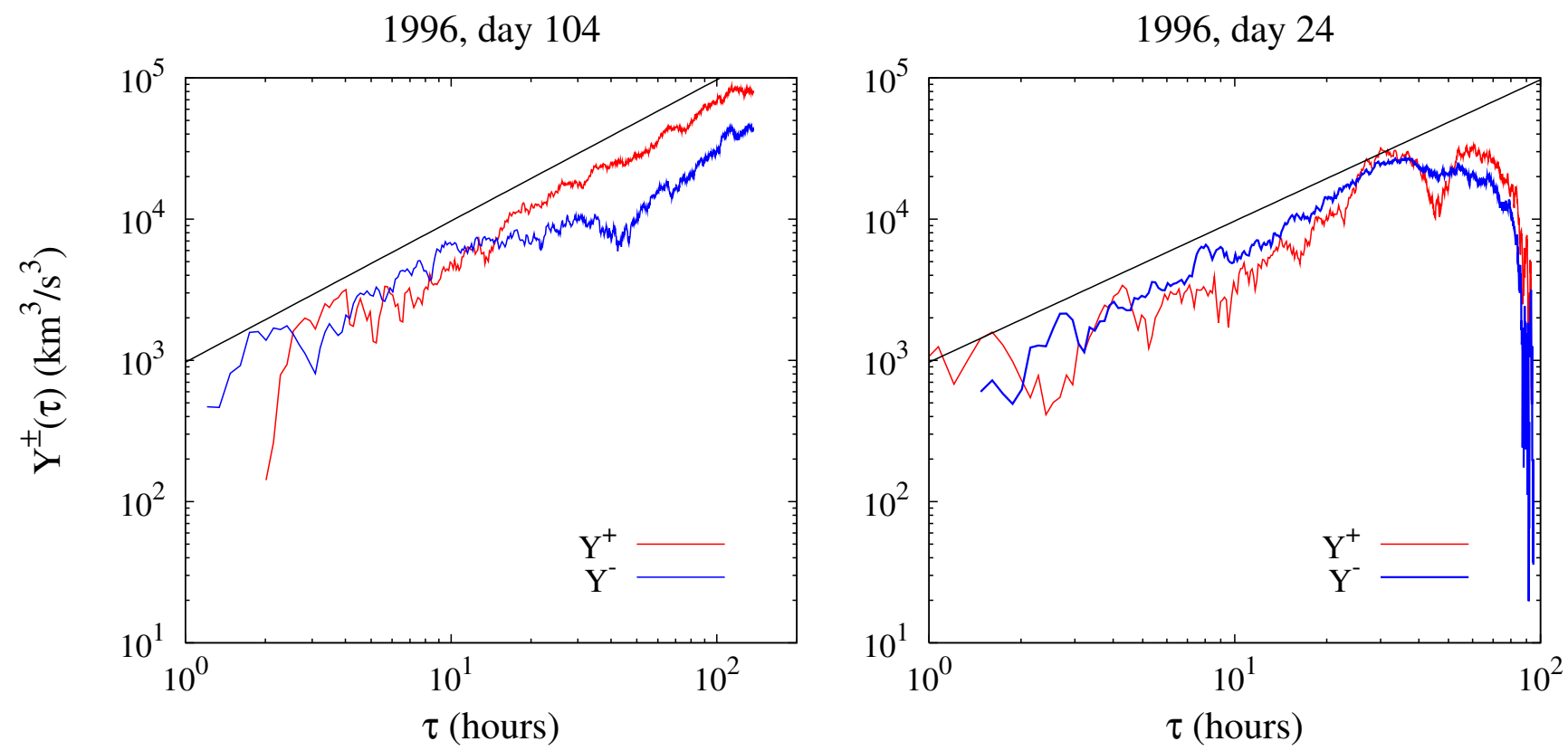

Figure 1: The scaling behaviour of both $Y^{ \pm}(\tau)$ as a function of the scale $\tau$ for two different periods we examined. The full black line shows a linear scaling law to guide the eye. 
Ulysses, 1996, hourly means

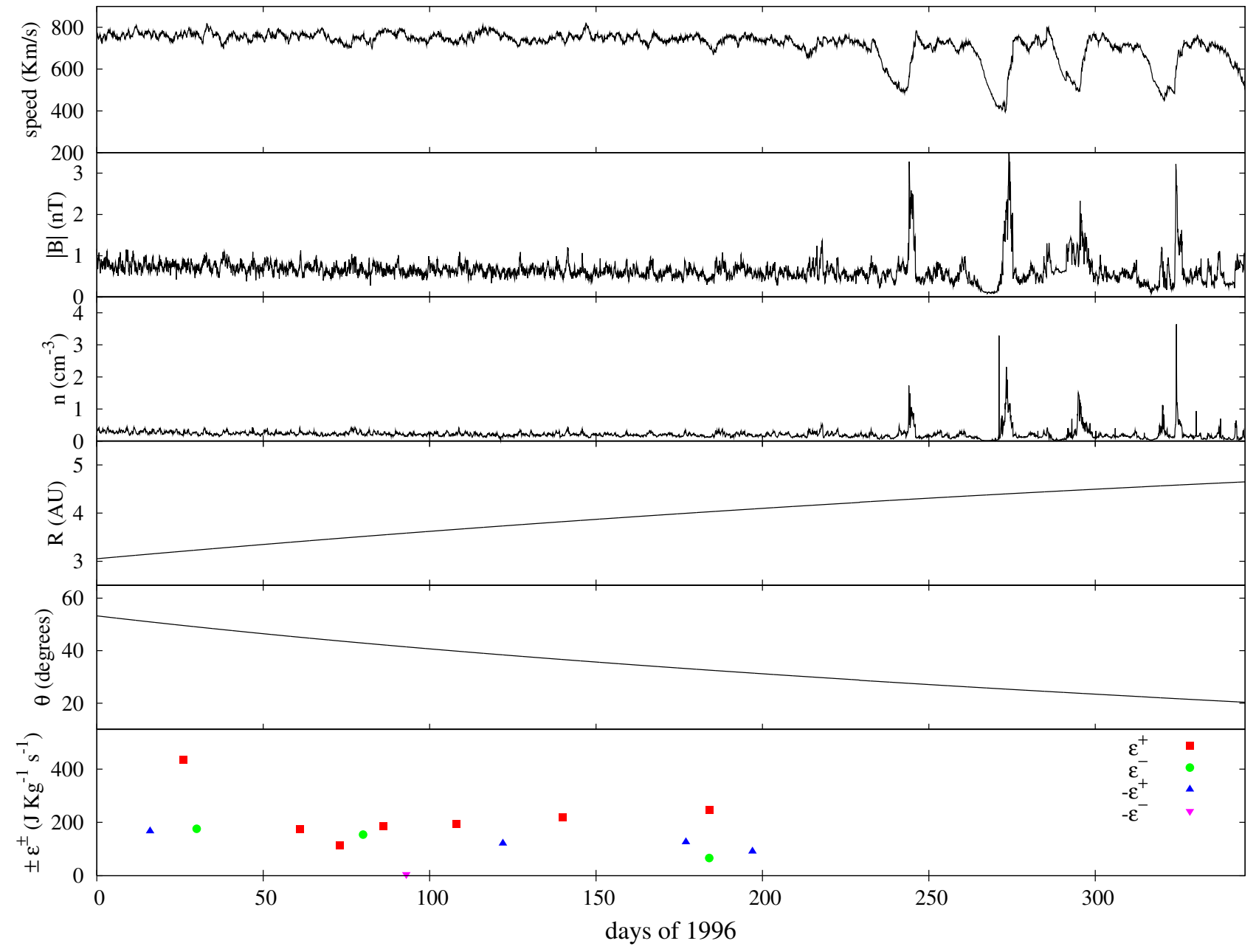

Figure 2: Hourly averaged quantities are represented as a function of the flight time of Ulysses. The top panels represent respectively the solar wind speed, the magnitude of the magnetic field, the particle density, the distance from the sun and the heliolatitude angle. In the bottom panel the values of $\epsilon^{ \pm}$, calculated through a fit with the function (3) during the periods where a clear linear scaling exists, are reported. 

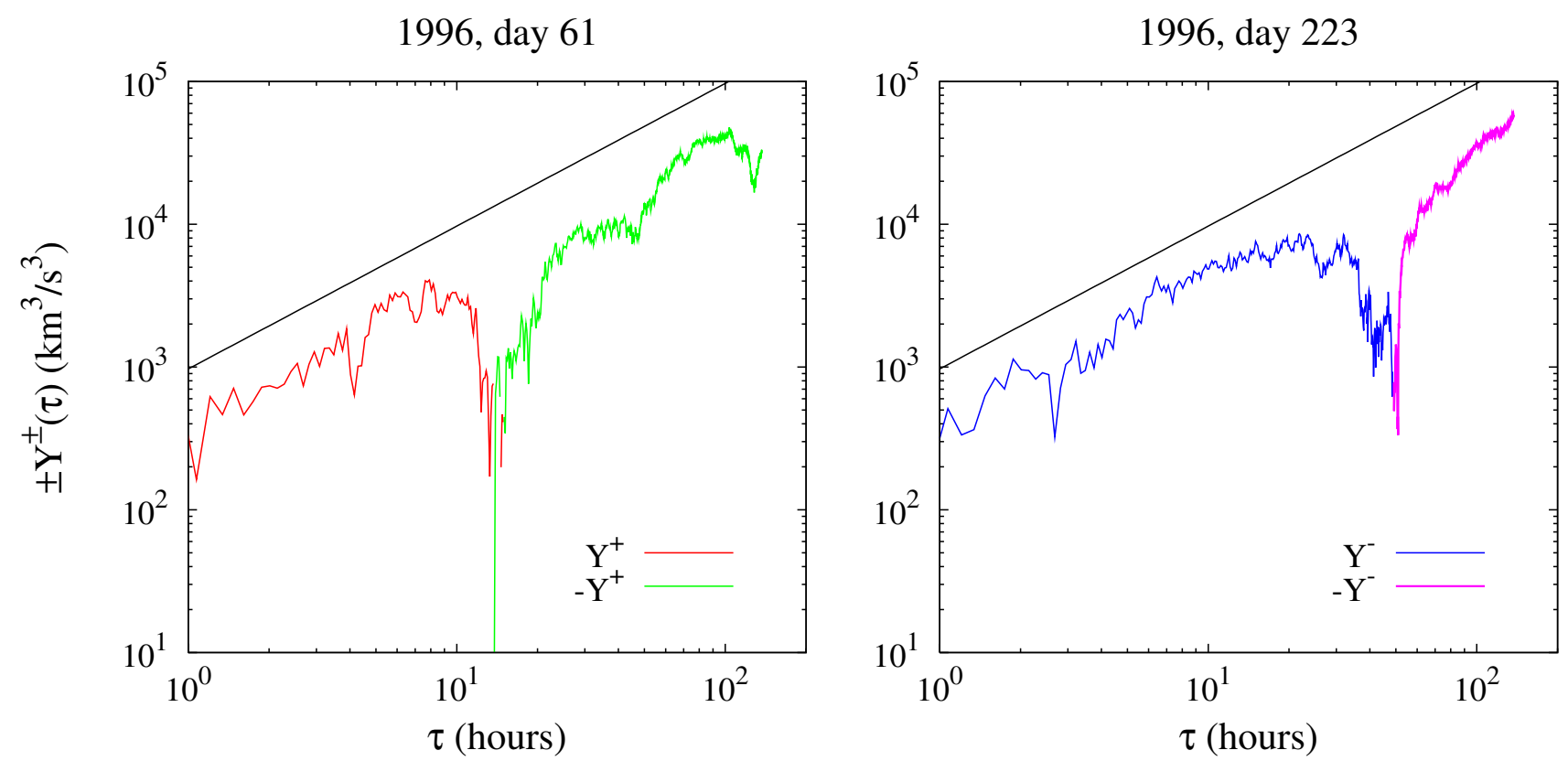

Figure 3: The scaling behaviour of $Y^{ \pm}(\tau)$ as a function of the time scale $\tau$ for two different periods we examined. Different colours of the curves refer to positive and negative values of the mixed structure functions $Y^{ \pm}(\tau)$ and thus of $\epsilon^{ \pm}$. The full black line correspond to a linear scaling law. 